\title{
ISO Tolerancing of hyperstatic mechanical systems with deformation control
}

\author{
Oussama ROUETBI ${ }^{1,2^{*}}$, Laurent PIERRE ${ }^{1}$, Bernard ANSELMETTI ${ }^{1}$ and Henri DENOIX ${ }^{2}$ \\ ${ }^{1}$ LURPA, ENS Cachan, Univ Paris Sud, Université Paris-Saclay, 61 avenue du président Wilson, F-94235 Cachan France \\ ${ }^{2}$ Etudes et Productions Schlumberger, 92140 Clamart, France \\ * Corresponding author. Tel.: +33664274719; fax: +0-000-000-0000. E-mail address: oussama.rouetbi@ens-cachan.fr
}

\begin{abstract}
The functional tolerancing of hyperstatic mechanisms provides contractual documents established following the ISO tolerancing. The tolerancing methodologies consider that the mechanism is infinitely rigid. These mechanisms impose tight clearances to ensure the functional requirements and parts fittability. The proposed methodology consists in developing a mechanical model relating the tolerances obtained by traditional methods of geometrical tolerancing and the parts deformability to define the tolerance values of the geometrical specifications. The first step is to define the geometrical specifications with ISO tolerancing. The fittability between two parts in contact requires maximum material conditions. The functional requirements employ least material condition. The second step consists in defining the capacity of parts to deform taking the tolerance values into account. A mechanical model is described relating the parts deformability to the tolerances to guaranty the conformity of the functional requirements and assembly parts fittability. As a validation example, the proposed methodology is used on a hyperstatic mechanism composed of two subassemblies: an outer tube and a shaft made of several assembled sections.
\end{abstract}

Keywords: ISO tolerancing; hyperstatic mechanism; deformation; maximum and least material requirements; mechanical model.

\section{Introduction}

In a classic tolerancing approach, the parts' defects are compensated by the clearances to guaranty the assembly requirements. Hyperstatic assemblies impose tight clearances. The defects are then compensated by certain parts' deformations. In the academic works involving geometrical tolerancing and deformation, the deviations are calculated at nominal dimensions of the CAD model. They are then added to the accumulated tolerances to verify the functional requirements (1).

In the case of hyperstatic mechanisms, in order to fit assembly parts, the deformations depend on the geometrical defects of parts. The determination of the accumulated tolerances is therefore necessary to calculate the forces to be applied and the deformation of the parts in the worst case. It is on the assembly so deformed that the different functional requirements can be verified.

This methodology is developed in this paper. Firstly, a defined mechanism is toleranced as an assembly of rigid parts to understand the assembly process difficulties and to guaranty the functional requirements. The next step relies on the capacity of parts to deform to integrate it into the tolerancing approach by building a mechanical model (finite elements analysis, beam...) as done in (2), (3) and (4).

To validate the proposed methodology, it is applied on an assembly inspired from a mechanism used by petroleum industries.

\section{Proposed methodology}

The logogram in Figure 1 shows the proposed methodology. This methodology exploits the deformability of parts composing a hyperstatic assembly to ensure the assembly and functional requirements. In order to optimise the tolerances, the main idea is to determine a mechanical model. The latter gives a mathematical relation between necessary forces to fit all the assembly parts and the tolerance values considering interferences between parts. Several studies have been considering contact interaction in tolerancing analysis (5), (6) and (7). 


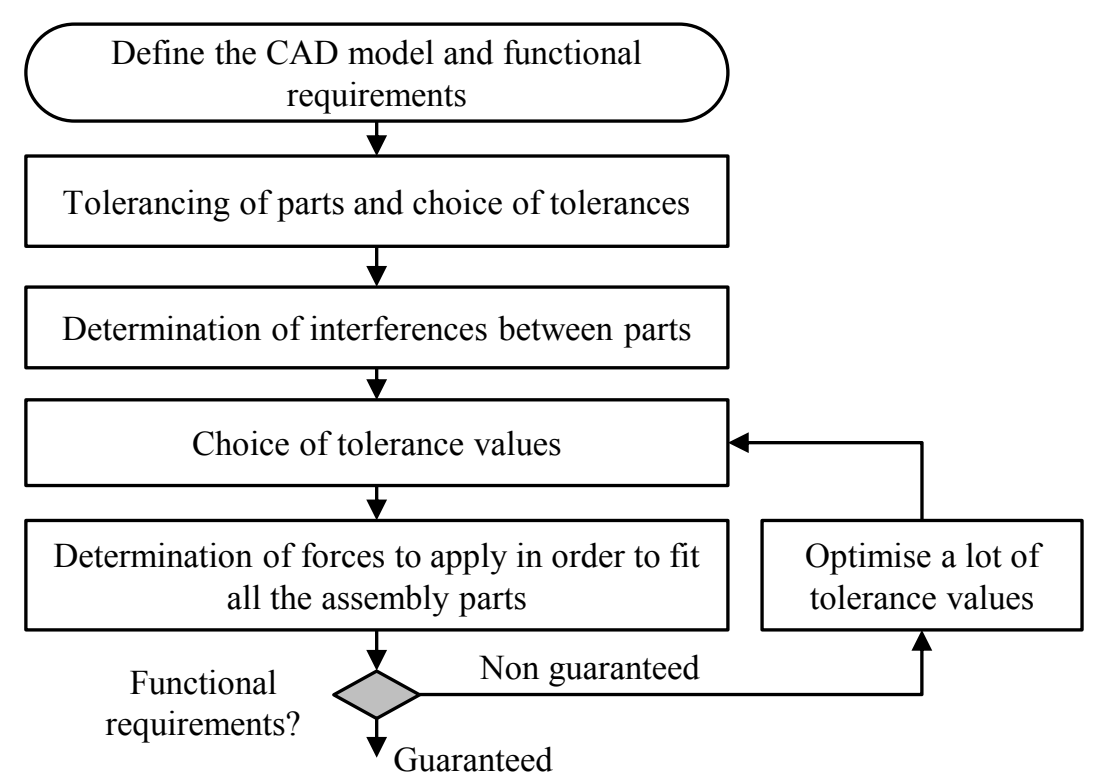

Fig. 1. Proposed methodology logogram.

\subsection{Tolerancing approach of rigid parts}

Generally, a designer tends to initially create a CAD model composed by parts with perfect shape at nominal dimensions. This model does not take into account the manufacturing or the assembly processes that introduce geometrical defects on the mechanism. The geometrical tolerancing ensures the fittability of parts and the functional requirements. In that purpose, the designer should specify geometrical specifications related to the assembly process and the functional requirements. The tolerancing approach is divided into three steps: tolerancing the parts in contact, tolerancing the assembly of parts and the transfer of functional requirements.

To ensure the assembly requirements of parts in contact, the designer should define the assembly process giving the order of the parts positioning and the adjustments operations. The assembly requirements can be performed using CLIC method (8) (French acronym for "Location Tolerancing with Contact Influence") developed by B. Anselmetti according to ISO tolerancing (9) with maximum material requirement (10). The geometric specifications are defined with respect to the assembly requirements guarantying the fittability of parts in contact. In the case of positioning subassemblies in contact, a transfer of the assembly requirements must be applied to each part. Various mathematical models allow calculating dimensioning chains like analysis lines method (8), the behaviour laws (11) and assembly conditions with polytopes (12).

Finally, to define the functional tolerancing, the designer needs to specify the operating conditions of the mechanism. The transfer of functional requirements is carried out using the same tools as for the transfer of assembly requirements. The tolerancing analysis will provide mathematical relations resolved by the analysis lines in Quick GPS (13), polytopes (14), deviation domains (15), T-map (16)... These conditions generate geometrical specifications with reference systems at least material requirements (10).

During these different steps, the designer can adjust the tolerances respecting various constraints including manufacturability, costs, etc.

\subsection{Tolerancing approach considering deformable parts}

If the various requirements (assembly, functional requirements) cannot be simultaneously respected, the proposed method can be applied taking the parts deformations into account. This method consists in determining a 
mechanical model giving a mathematical relation between necessary forces to fit all the assembly parts and the tolerance values.

The designer studies the capacity of the different parts to be deformed. For each part, the deformability depends on the size, the shape and the used material. The deformability behaviour can be different in one direction and not in the others. The next step is to build a mechanical model at nominal dimensions, respecting the shapes and the functional or assembly requirements. For example, a beam model can be used rather than finite elements model for slim parts. In boundary conditions, the effect of geometrical variations of manufacturing and assembly processes is introduced at the beam model as displacements. For a finite element model, some displacements of nodes are imposed. Finally, the designer proposes a geometrical model, taking the effect of geometrical defects and the parts deformability into account.

The last step consists in optimising a lot of tolerances which aims at maximizing them, i.e. minimizing their cost by increasing their values while respecting the functional requirements and the maximum mechanical stress in and between parts, etc.

\section{Application}

\subsection{Description of mechanism}

The studied mechanism is a hyperstatic assembly composed of two subassemblies: an outer tube and a shaft made of several assembled sections (see Figure 2). The shaft is composed mainly of 4 slim parts separated by seals supports.

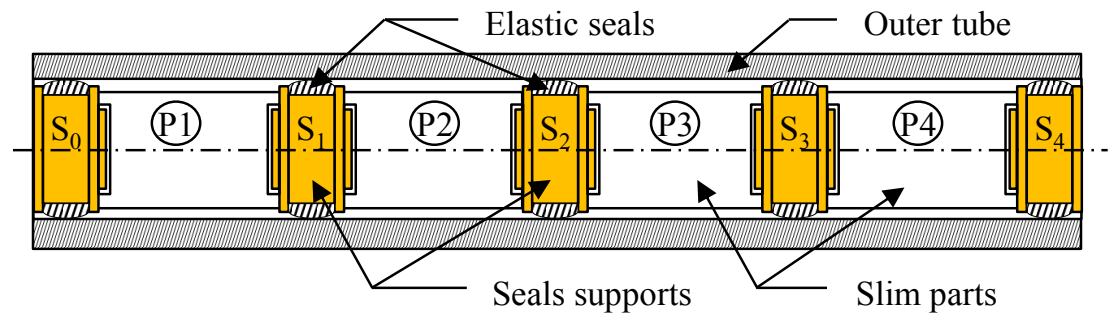

Fig. 2. Hyperstatic assembly of a multi-components shaft in an outer tube

For the proper functioning of the mechanism, contacts must not occur between metallic parts of the shaft and the tube. To guarantee the non-contact metal to metal, the shaft guidance is ensured using elastic seals, which should be mounted with some squeeze.

Since the mechanism is symmetric, the assembly process is described as follow: the shaft assembly can start from any of the two end-seal supports $\left(\mathrm{S}_{0}\right.$ or $\mathrm{S}_{4}$ shown in Figure 2). Each seal support or slim part is positioned with the previous part by planar contact and short cylinder, and maintained in position using four screws. Once the shaft is assembled, it is inserted into the outer tube by pulling it.

The difficulty at this level is to compensate the geometrical defects of the shaft to be able to insert it into the outer tube while maintaining permanent contacts at elastic seals levels (see Figure 3). To overcome the assembly difficulties, shaft parts and elastic seals deformations are required. The elastic seals squeezes involve contact forces and intense friction with the outer tube during insertion.

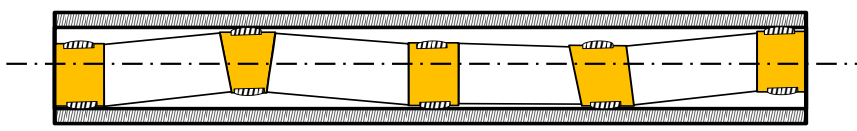

Fig. 3. Example of straightness default by geometrical defects 


\subsection{Functional tolerancing of rigid parts}

The functional tolerancing is performed with CLIC method (8). The assembly requirement between shaft parts is described by geometrical specifications with (1) asterisk in Figure 4. The transfer of shaft straightness is guaranteed by specifications with (2) asterisk in Figure 4. To guarantee the non-detachment of the seals, their supporting surface is taken as reference on seals supports to locate the geometrical specifications (reference F in Figure 4.b).

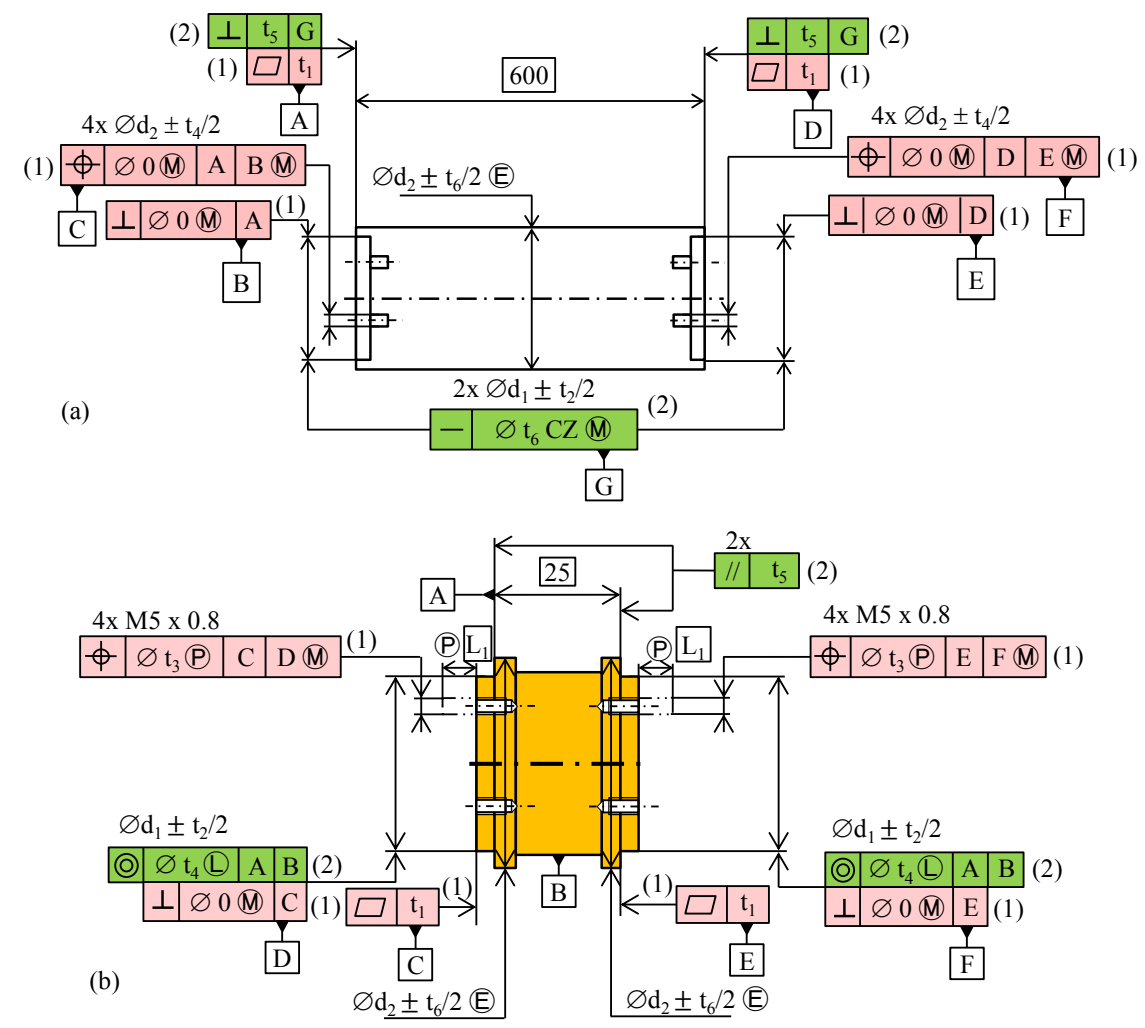

Fig. 4. (a) Geometrical specifications of slim part (b) Geometrical specifications of seal support

For the case of the outer tube, three geometrical specifications (see Figure 5) are proposed to ensure the fittability (straightness at maximum material), contact maintaining (straightness at least material) and evenly distributed contacts at elastic seals levels (cylindricity and envelope requirement).

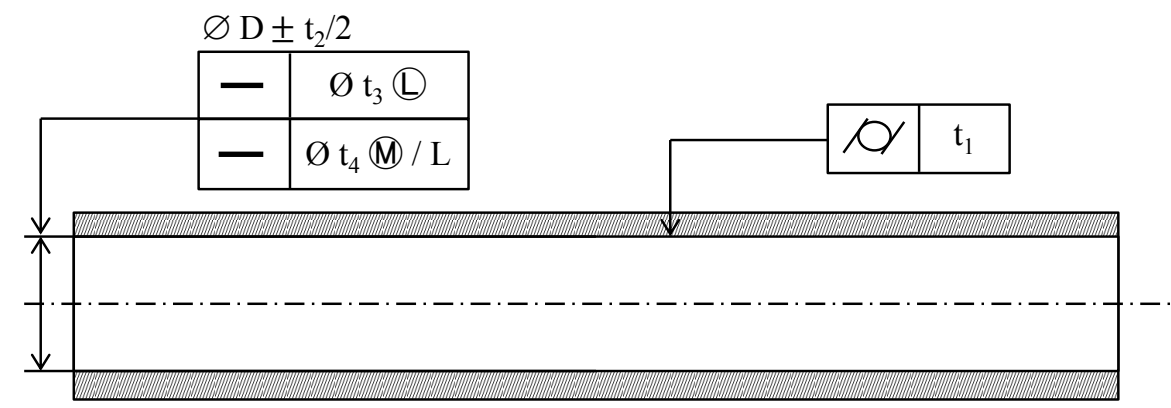

Fig. 5. Geometrical specifications of slim parts, seal support and the outer tube

This paper examines mainly the straightness of the shaft at the final step of insertion into the outer tube to find a mathematical relation between necessary forces to fit all the assembly parts and the tolerance values. 
Firstly, the tolerancing analysis was performed by the analysis lines method (8) to detect the impact of geometrical defects and understand the worst cases configurations for the straightness of the shaft maximising the insertion forces. The result of this study is that the straightness is mainly affected by the clearances and the angular deviations on the junctions between the seals supports and the slim parts as shown in Figure 6.

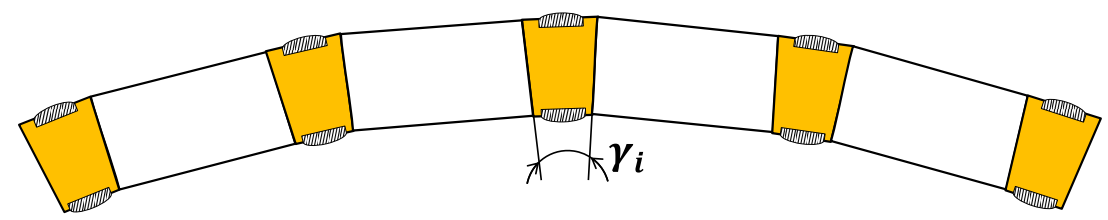

Fig. 6. Worst case configuration with planar deviations

\subsection{Assembly requirements of deformable parts}

To guaranty the insertion according to analysis lines method, tight tolerance values are generated. For that purpose, and regarding the shape of the slim parts, a beam model can be built. The insertion force is defined as a sum of influences of different tolerances in the form:

$$
F_{\text {insertion }}=\sum c_{i} \cdot t_{i}
$$

The shaft is assimilated to a continuous and deformable beam with different sections for each slim part. The shaft is inserted into the outer tube supposed infinitely rigid with punctual elastic contacts using springs. The geometrical defects of the outer tube will be transposed on the shaft. As a result, the studied mechanism can be simplified into a beam laid on elastic unilateral contacts using springs (see Figure 7). The deviation of each junction between each slim part and adjacent seal supports, created by the clearances and the angular deviations, will be introduced in the beam model in boundary conditions as displacements noted $\delta_{i}$ (see Figure 7). The maximum value of $\delta_{i}$ is evaluated by the analysis line method and taking as reference the line passing through the centers of $\mathrm{S}_{0}$ and $\mathrm{S}_{1}$ :

$$
\delta_{i \max }=\sum k_{j} \cdot t_{j}
$$

The mechanism is studied as a beam laid on $\mathrm{N}$ elastic unilateral contacts (see Figure 7).

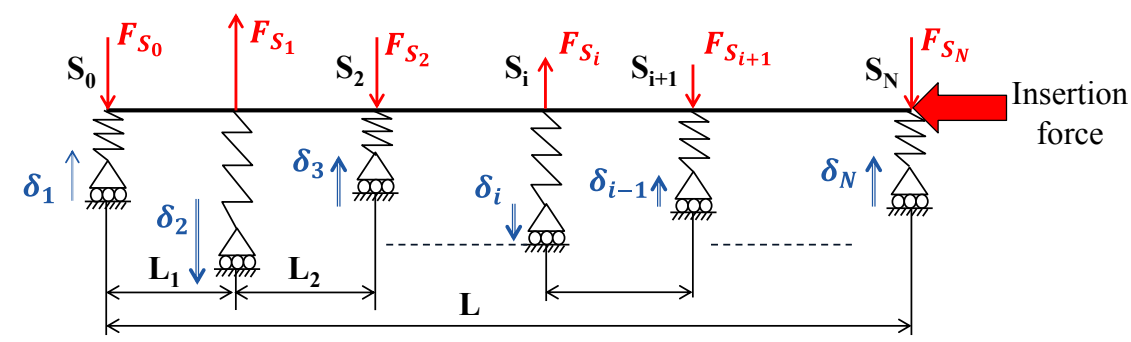

Fig. 7. 2D Beam model

An energetic method applied on beam theory determinates a matrix $\mathbf{T}$ respecting the static balance of the beam and springs. The springs release their energies to the beam creating its deformation. The equation (3) is established giving the relation between the vector of contact forces $\mathbf{F}_{\mathbf{S}}$ and the boundary conditions $\boldsymbol{\delta}$.

$$
\mathbf{F}=\mathbf{T} \times \boldsymbol{\delta}
$$


This methodology was applied putting the shaft in the worst case as described by the analysis lines method in Figure 6 . The insertion force has the form of equation (4) by straighten the shaft inside the outer tube, where $\mu$ is the friction coefficient between each seal and the outer tube. $\mathbf{f}$ is the row matrix of friction.

$$
F_{\text {insertion }}=\sum \mu \cdot F_{S_{i}}=\mathbf{f} \times \mathbf{F}_{\mathbf{S}}=\mathbf{f} \times \mathbf{T} \times \boldsymbol{\delta}
$$

The worst case configuration according to analysis lines method gives a reduced insertion force could be not the worst case for the insertion force. For that reason, the research of the worst case configurations were integrated to the beam model. By generating 3D geometrical deviations, the beam model, as it was described, is used to calculate the insertion force of all possible configurations (see Figure 8).

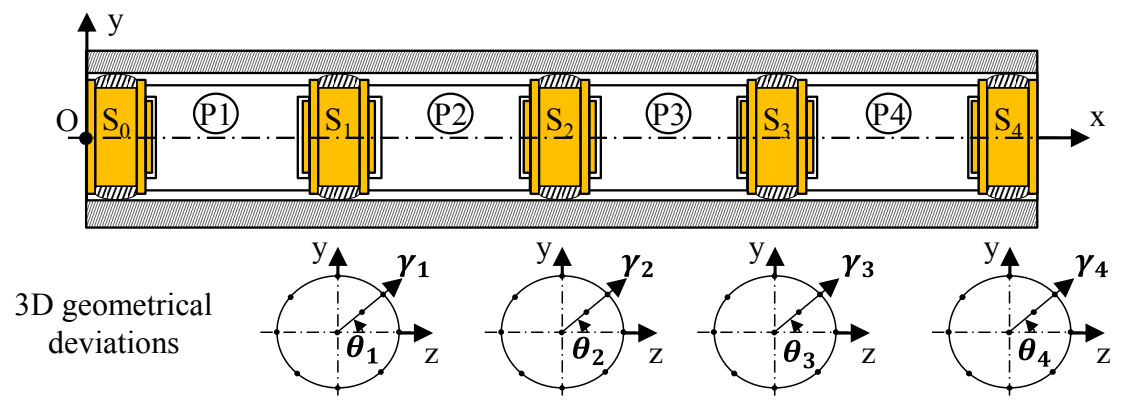

Fig. 8. 3D Geometrical deviations

As shown in Figure 8, $\theta_{i}$ and $\gamma_{i}$ represent the orientation defaults in the junctions between seals supports and the slim parts. By this approach, the worst case configuration occurs when the all the $\gamma$ values are maximized and the $\theta$ values are alternatively between zero and $\pi$ from one junction to the next. The shaft takes the form of repeated "W" in Oxy and repeated "W" in Oyz. At this shape, the insertion force is estimated to 2.5 times the insertion force obtained with planar model (see Figure 6).

The last step of the proposed methodology is to optimise the different tolerance values. It aims to find a compromise between minimizing the cost by increasing tolerances while respecting a maximum insertion force.

$$
(\mathbf{f} \times \mathbf{T} \times \boldsymbol{\delta}) \leq \text { Finsertion }_{\max }
$$

\section{Conclusions}

This paper presents a methodology that provides a mathematical model in order to optimise the tolerance values, relating them to the necessary forces to fit all assembly parts. The encountered geometrical defects, on a mechanism, include the variability of the manufacturing and the assembly processes, as well as the mechanical model (finite elements analysis, beam model...) taking into account the parts' deformability. This methodology is illustrated to estimate the necessary forces to assemble the mechanisms used by petroleum industries.

This methodology optimises only tolerance values rather than proposing geometrical specifications. One of the future perspectives for this work is to take into account the parts' capacity to deform at the level of the transfer of functional requirements. The tolerancing analysis methodology used in this article can be applied to other types of mechanical systems. 


\section{References}

1. J. Stuppy and H. Meerkamm, Tolerance analysis of a crank mechanism by taking into account different kinds of deviation, in $11^{\text {th }}$ CIRP CAT Conference, Annecy, France, March 2009.

2. G. Cid, F. Thiébaut and P. Bourdet, Taking the deformation into account for components' tolerancing, in 5th Conference on IDMME, Bath, UK, April 2004

3. G. Cid, Etablissement des relations de comportement de mécanismes avec prise en compte des écarts géométriques et des souplesses des composants, PhD thesis, ENS Cachan, December 2005.

4. I. A. Manarvi and N. P. Juster, Framework of an integrated tolerance synthesis model and using FE simulation as a virtual tool for tolerance allocation in assembly design, Journal of Materials Processing Technology, vol. 150, no. 1-2, pp. 182-193, 2004.

5. K. Xie, L. Wells, J. A. Camelio and B. D. Youn, Variation Propagation Analysis on Compliant Assemblies Considering Contact Interaction, Journal of Manufacturing Science and Engineering, ASME, vol. 129, no. 5, pp. 934-942, October 2007.

6. L. Pierre, D. Teissandier and J.-P. Nadeau, Integration of thermomechanical strains into tolerancing analysis, International Journal on Interactive Design and Manufacturing, vol. 3, no. 4, pp. 247-263, 2009.

7. S. J. Hu and J. Camelio, Modelling and control of compliant assembly, CIRP Annals - Manufacturing Technology, vol. 55, no. 1, pp. 19-22, 2006.

8. B. Anselmetti, Generation of functional tolerancing based on positioning features, Computer-Aided Design, vol. 38, no. 8, pp. 902-919, August 2006.

9. ISO1101:2013, Geometrical Product Specifications (GPS), Geometrical tolerancing, Tolerances of form, orientation, location and run-out, 2013.

10. ISO2692:2014, Geometrical product specifications (GPS) - Geometrical tolerancing - Maximum material requirement (MMR), least material requirement (LMR) and reciprocity requirement (RPR), 2014.

11. E. Ballot, Lois de comportement géométrique des mécanismes pour le tolérancement, PhD thesis, Université de Nancy, 1995.

12. D. Teissandier, V. Delos and Y. Couetard, Operations on polytopes: application to tolerance analysis, Kluwer academic publisher, 1999, pp. 425-433.

13. B. Anselmetti, R. Chavanne, N. Anwer and J.-X. Yang, Quick GPS: A new CAT system for single-part tolerancing, ComputerAided Design, vol. 42, no. 9, pp. 768-780, 2010.

14. L. Pierre, D. Teissandier and J.-P. Nadeau, Variational tolerancing analysis taking thermomechanical strains into account: Application to a high pressure turbine, Elsevier, vol. 74, pp. 82-101, 2014

15. M. Giordano, S. Samper and J.-P. Petit, Tolerance analysis and synthesis by means of deviation domains, axisymmetric cases, in Models for Computer Aided Tolerancing in Design and Manufacturing, Springer, 2007, pp. 85-94.

16. J. K. Davidson, A. Mujezinović and J. J. Shah, A New Mathematical Model for Geometric Tolerances as Applied to Round Faces, Journal of Mechanical Design, vol. 124, no. 4, pp. 609-622, 2002. 